\title{
Investigación en industrias culturales: construcción bibliográfica y aproximaciones a su uso conceptual
}

DOI: https://doi.org/10.21158/01208160.n0.2020.2840

\author{
Luis David Rodríguez-Romero ${ }^{1}$ \\ luis.rodriguezr@uniagustiniana.edu.co \\ Universitaria Agustiniana \\ Oswaldo Ospina-Martínez ${ }^{2}$ \\ oswaldo.ospinam@uniagustiniana.edu.co \\ Universitaria Agustiniana
}

\section{Fecha de recepción:20 de mayo de 2020 \\ Fecha de aprobación: 10 de diciembre de 2020 \\ Fecha de aprobación: 12 de abril de 2021}

Cómo citar este artículo / To reference this article / Comment citer cet article / Para citar este artigo:

Rodríguez-Romero, L. D.; Ospina-Martínez, O. (2020). Investigación en industrias culturales: construcción bibliográfica y aproximaciones a su uso conceptual. Revista Escuela de Administración de Negocios, Edición especial 2020, 135 - 150. DOI: https://doi.org/10.21158/01208160.n0.2020.2840

\section{Resumen}

Este artículo presenta el uso del concepto industrias culturales como derrotero de procesos investigativos a través de una construcción bibliográfica. Inicia con una revisión literaria que abarca desde el año 2000 hasta el año 2018, mediante la exploración de 25 artículos de impacto, a nivel global, de las principales revistas indexadas dentro de la base de datos Scopus. Se trata de un estudio no experimental, de tipo exploratorio y conceptual que logró la identificación de temáticas asociadas a las investigaciones de este concepto y al reconocimiento de nuevos espacios de apropiación. Se reconoció la transformación de las industrias culturales de intermediarias a garantes de derechos, funcionando como catalizadoras de nuevos espacios de inclusión. Además, su investigación se ha centrado en poblaciones y grupos humanos específicos, lo que ha generado la inminente necesidad de mayor regulación del sector cultural, en la medida en que se presentan diferentes modos y formas de accionar, de tal forma que no existen estructuras definidas. Este artículo se plantea como una primera fase de investigación que tomó como punto de inicio el propio concepto de industrias culturales, por ser el entorno general para la búsqueda de información, con el fin de ampliarlo a la totalidad del sector cultural en futuras investigaciones que, además, impacten y generen resultados específicos dentro de cada uno de los subsectores que hacen parte del ecosistema creativo y cultural.

Palabras clave: industrias culturales; concepto de industrias culturales; producción científica; revistas indexadas; revisión bibliográfica.

\footnotetext{
1 Profesional en Negocios Internacionales en formación de la Universitaria Agustiniana. Integrante del semillero de investigación en Negocios Internacionales - SINI. Universitaria Agustiniana. ORCID: https://orcid.org/0000-0002-2256-6799

${ }^{2}$ Magíster en Psicología del Consumidor - Fundación Universitaria Konrad Lorenz. Especialista en Psicología del Consumidor Fundación Universitaria Konrad Lorenz. Especialista en Gerencia de Mercadeo - Universidad de Bogotá Jorge Tadeo Lozano. Administrador de Empresas - Universidad Antonio Nariño. ORCID: https://orcid.org/0000-0003-3889-5973
} 


\section{Research in cultural industries: $\longrightarrow$ bibliographic construction and approaches to its conceptual use}

\section{Abstract}

This article presents the use of the concept of cultural industries as a path for research processes through a bibliographic construction. It begins with a literature review that covers from 2000 to 2018, by means of the exploration of 25 impact articles, at a global level, from the main journals indexed in the Scopus database. This is a non-experimental, exploratory, and conceptual study that achieved the identification of topics that are associated to the research on this concept, and to the recognition of new spaces of appropriation. We recognized the transformation of cultural industries, from intermediaries into duty bearers, functioning as catalysts of new spaces of inclusion. In addition, the research has focused on specific populations and human groups, which has generated the imminent need for greater regulation of the cultural sector, inasmuch as there are different ways and forms of action, so there are no defined structures. This article is proposed as a first phase of research that took the very concept of cultural industries as a starting point, since it is the general environment for the search of information, with the aim of extending this information to the entire cultural sector in a future research that will also impact and generate specific results within each of the subsectors that are part of the creative and cultural ecosystem.

Keywords: cultural industries; concept of cultural industries; scientific production; indexed journals; literature review.

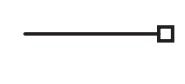

\section{Pesquisa em indústrias culturais: construção bibliográfica e abordagens para seu uso conceitual}

\section{Resumo}

Este artigo apresenta a utilização do conceito de indústrias culturais como rumo dos processos de pesquisa por meio de uma construção bibliográfica. Inicia com uma revisão literária que abrange o ano de 2000 até o ano de 2018, explorando 25 artigos de impacto, a nível mundial, das principais revistas indexadas na base de dados Scopus. É um estudo não experimental, exploratório e conceitual que conseguiu identificar temas associados às investigações deste conceito e ao reconhecimento de novos espaços de apropriação. Reconheceuse a transformação das indústrias culturais de intermediárias em garantes de direitos, funcionando como catalisadores de novos espaços de inclusão. Além disso, sua pesquisa tem se voltado para populações e grupos humanos específicos, o que tem gerado a necessidade iminente de maior regulação do setor cultural, na medida em que se apresentam diferentes modos e formas de ação, de tal maneira, que não existem estruturas definidas. Este artigo é proposto como uma primeira fase de pesquisa que tomou como ponto de partida o próprio conceito de indústrias culturais, por ser o ambiente geral de busca de informação, com a finalidade de estendê-lo a todo o setor cultural em pesquisas futuras e, além disso, possam impactar e gerar resultados específicos dentro de cada um dos subsetores que fazem parte do ecossistema criativo e cultural.

Palavras-chave: indústrias culturais; conceito de indústrias culturais; produção científica; revistas indexadas; revisão bibliográfica. 


\section{Résumé}

\section{Construction bibliographique et approches conceptuelles de l'industrie culturelle}

Cet article présente l'utilisation du concept d'industrie culturelle comme un processus d'investigation à partir d'une construction bibliographique. Nous réaliserons d'abord une analyse de la littérature recouvrant les années 2000 à 2018 en explorant 25 articles majeurs parus dans les principales revues répertoriées dans la base de données Scopus. Il s'agit d'une étude non expérimentale, exploratoire et conceptuelle qui identifie les thèmes associés aux recherches sur ce concept et à la reconnaissance de nouveaux espaces d'appropriation. Les industries culturelles se sont transformées et sont passées du rang d'intermédiaires à celui de garantes des droits en fonctionnant comme catalyseurs pour de nouveaux espaces d'inclusion. Par ailleurs, les recherches se sont concentrées sur des populations et des groupes humains spécifiques, créant le besoin prégnant d'une plus forte régulation du secteur culturel dans lequel sont menés différents modes et types d’actions afin de juguler la création de structures prédéfinies. Cet article n'est qu'une première phase d'investigation prenant comme point de départ le concept même d'industrie culturelle. Cette recherche d'information pourrait s'étendre à l'ensemble du secteur culturel lors de futures investigations qui impacteraient et génèreraient des résultats spécifiques au sein de chacun des sous-secteurs de l'écosystème de la création culturelle.

Mots-clés: industries culturelles; concept d'industries culturelles; production scientifique; revues indexées; passage en revue de la littérature. 


\section{Introducción}

as industrias culturales se caracterizan por
crear, producir y comercializar bienes y servicios culturales cargados de un valor inmaterial representativo; por lo que son parte inherente de la identidad de los pueblos. Los estudios e investigaciones sobre estas pueden considerase jóvenes, en la medida en que las industrias culturales han empezado a influir en el desarrollo de la economía mundial desde hace poco menos de 15 años, convirtiéndose en generadoras de espacios de integración social y de cooperación intrarregional, en cuanto garantes de derechos humanos y como catalizadoras de emprendimientos.

Lo anterior se explica si se tiene en cuenta el impacto que han generado de un tiempo para acá y que se logra identificar con el informe titulado Tiempos de cultura: el primer mapa mundial de las industrias culturales y creativas (2015), realizado por EY y encargado por la Confederación Internacional de Sociedades de Autores y Compositores (Cisac). En él se observa la manera cómo estas industrias aportan a la economía global de forma significativa, no solo en términos de ingresos, sino también de generación de empleo, desarrollo de las ciudades y beneficios que involucran a la cultura; ya sea mediante la creación de identidad, el reconocimiento social o el posicionamiento regional.

Las industrias culturales en el desarrollo global actual se han presentado como necesarias y aportantes relevantes en la integración social y en la vinculación laboral, ya que basan su actividad en estándares de habilidad y talento humano, uso de la creatividad y procesos de innovación: «El referirse a este tipo de industrias supone la ampliación del espectro original, debido a que mezcla lo económico, lo cultural y la formalización empresarial en un éxito inagotable» (Redondo-Méndez, 2019, p. 27).

Por esta razón se hace necesario reconocer los espacios de investigación y desarrollo de temáticas asociadas al sector cultural que sirvan de derrotero en futuros espacios no solo académicos, sino también gubernamentales y civiles, para lograr integrar esferas que impulsen el sector desde sus propias particularidades.

Este documento tiene como finalidad presentar la utilización del concepto de industrias culturales en revistas indexadas dentro de la base de datos Scopus, mediante una construcción de literatura, que tiene como base los artículos seleccionados con el descriptor industrias culturales a nivel mundial, en el periodo 2000 a 2018. Se trata de una aproximación al uso del concepto, en el marco de las investigaciones de mayor impacto en la base de datos seleccionada, con el fin de identificar los grupos de temas de mayor impacto bajo este concepto/descriptor, para así lograr establecer una guía para futuras investigaciones asociadas a este tópico.

Si bien se trata de una selección inicial, debido a que existen mayores referencias al tema cultural a partir de descriptores diversos, permite una identificación de las investigaciones que han desarrollado el tema, desde su óptica más general, y que han desarrollado temáticas en torno al sector cultural; esto permite reconocer transformaciones, evoluciones o construcciones en torno al concepto seleccionado.

Se debe mencionar que es una representación de tipo conceptual y exploratorio que se ubica en una temporalidad y en una espacialidad específica. No pretende ser una única revisión, sino establecer la relación que se ha presentado, en esta línea temática de las industrias culturales, entre procesos investigativos y materializaciones de su uso.

También es importante resaltar que se trató de la primera etapa de la primera fase de investigación correspondiente al ecosistema creativo del sector cultural, lo que permite un acercamiento a la base estructural de los estudios que se han desarrollado con esta temática y permiten plantear una agenda de investigaciones futura para Colombia y Latinoamérica. 
Las fases posteriores de la investigación han representado el reconocimiento de nuevos escenarios para la academia con respecto a la potencialidad del sector cultural desde procesos de indagación hasta la materialización de su utilización y el aprovechamiento de sus ventajas; esto a partir de la ampliación de la búsqueda por descriptores que abarcan los subsectores y el contexto de la cultura.

\section{Metodología}

a metodología utilizada corresponde, primero, a
un estudio no experimental, en cuanto se llevó a cabo la observación de los procesos investigativos ya existentes. Su diseño fue de tipo longitudinal, si se tiene en cuenta que el periodo de tiempo abarcado permite identificar los cambios y las transformaciones realizadas a partir de la primera aparición en la base de datos seleccionada.

Para esta primera fase se realizó una revisión literaria, mediante el descriptor industrias culturales y la aplicación de filtros en años -2000-2018_, en áreas temáticas - extrayendo lo concerniente a ciencias exactas o de la salud-y documentos que sean de revistas. Una vez se estableció la cantidad de documentos que aplicaban el filtro, se realizó un barrido para determinar, según el título, qué revista ha publicado al menos una vez un artículo con relación a las ICC.

Además, de esta forma, también con ayuda de la base de datos mencionada, revisaron las características propias de las revistas: país de origen, categoría de las ICC, número de documentos publicados y citas de estos documentos durante el periodo investigado, número de artículos que estudian las ICC del 20002018 y los indicadores de Cite Score y Cuartil.
Se trató de un estudio bibliográfico de tipo expositivo e informativo en el que se pretende recrear el contexto investigativo sobre el tema, a través de «revisar, criticar, y sintetizar la literatura» (Torraco, 2005, 42). Esta es una primera fase de la investigación que tiene como propósito la construcción completa de la revisión bibliográfica de los tópicos que hacen parte de las investigaciones en economía creativa. El alcance planteado es descriptivo, en la medida en que se basa en una investigación conceptual y documental, con el fin de reconocer el uso del concepto y su manifestación en los métodos para la investigación.

De esta manera, el estudio construye una estructura organizativa y conceptual que permite un acercamiento al estado del arte de las industrias culturales - con este descriptor como primer pasoque demuestra el uso del concepto y un primer acercamiento a la identificación de futuros estudios que generen procesos de desarrollo económico y social. El artículo se estructura en cuatro partes: introducción, metodología, resultados y discusión, y conclusiones, las cuales complementan y permiten la identificación de la transformación del concepto a través de las investigaciones. 


\section{Resultados}

\subsection{Conceptualización de las industrias culturales}

El propio concepto de las industrias culturales ha manifestado un desarrollo un tanto tardío, pero bastante cargado de elementos propios de la economía, el comercio y la cultura. Si se quiere, un punto inicial tuvo que ver con su aparición en la Escuela de Frankfurt, en manos de Theodore Adorno y Max Horkheimer (1988), quienes manifestaron su existencia a partir de la transformación de bienes de tipo cultural en objetos comerciales para satisfacer cierto tipo de necesidades de tipo consumista: «Consideran al arte como rupturista, como elemento que permite la discontinuidad y el extrañamiento, es decir, una discordia al menos con la experiencia, con la vida capitalista burguesa» (Follari, 2003).

Sin embargo, y a partir de su recurrencia, este concepto se ha transformado hasta el punto de relacionarlo de forma directa con la economía y no verlo como aspecto diferenciado sino como elemento integrador, empezando en el Reino Unido a finales de la década de los noventa. El representante de la noción que vincula a la cultura con la economía y permite hablar de manera específica de las industrias culturales es John Howkins (2001), quien creó el concepto de economía creativa en cuanto unión entre la creatividad y la dimensión económica, atravesada por la innovación y el talento del ser humano.

Actualmente se puede decir que

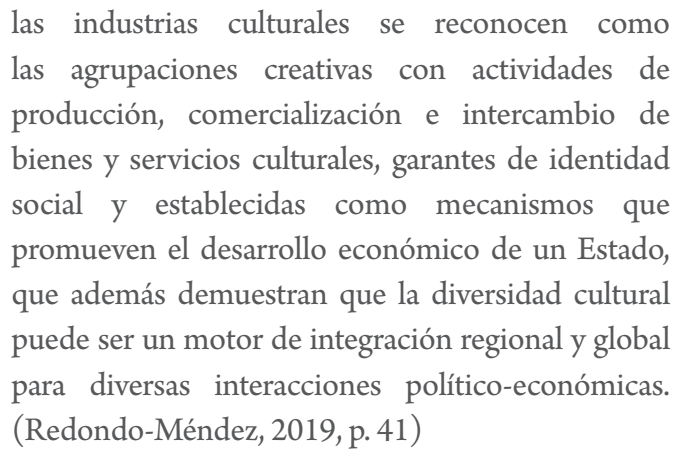

El estudio de las industrias culturales ha representado a nivel global la necesidad de materializar su funcionalidad y su reconocimiento como área interdisciplinar relevante para el desarrollo de un Estado. Esto se ha manifestado en sus múltiples funcionalidades dentro de una sociedad, al ser garantes de crecimiento económico, de inclusión social, de defensa de derechos humanos y de generación de nuevas oportunidades de vinculación laboral.

\subsection{Aproximaciones a su uso conceptual}

El proceso de revisión arroja un total de 25 artículos bajo el descriptor industrias culturales, en el periodo 2000-2018, una cifra que, aun con un descriptor general, es baja; igualmente, se demuestra que el $38 \%$ de las publicaciones encontradas corresponden al año 2018, mientras que el $3 \%$ corresponde al 2000. En la tabla 1 se presentan los resultados de la revisión general. 
Tabla 1. Artículos-descriptor industrias culturales desde el año 2000 hasta el 2018

\begin{tabular}{|c|c|c|c|c|}
\hline$\#$ & Artículo & Autor & Año & Journal \\
\hline 1 & «Innovaciones en política cultural y desarrollo en América Latina» & Yúdice, G. & 2018 & $\begin{array}{l}\text { International } \\
\text { Journal of } \\
\text { Cultural Policy }\end{array}$ \\
\hline 2 & $\begin{array}{l}\text { «a invención del cocacolo: <americanización> y diferenciación social en } \\
\text { Bogotá en la década de } 1950 »\end{array}$ & Flórez-Malagón & 2018 & $\begin{array}{l}\text { Canadian Journal of Latin } \\
\text { American and Caribbean } \\
\text { Studies }\end{array}$ \\
\hline 3 & $\begin{array}{l}\text { «Industrias creativas y culturales: estudio desde el enfoque de la gestión del } \\
\text { conocimiento» }\end{array}$ & $\begin{array}{l}\text { Blanco-Valbuena, } \\
\text { Bernal-Torres, } \\
\text { Camacho y Díaz-Olaya }\end{array}$ & 2018 & Información Tecnológica \\
\hline 4 & $\begin{array}{l}\text { «Perspectivas investigativas en el estudio de las industrias culturales y } \\
\text { creativas» }\end{array}$ & $\begin{array}{l}\text { Avendaño, Arias y } \\
\text { Arias }\end{array}$ & 2018 & Kepes \\
\hline 5 & $\begin{array}{l}\text { «Industrias culturales y composición de los personajes en las series de } \\
\text { animación infantil emitidas en España» }\end{array}$ & $\begin{array}{l}\text { Ortega-Mohedano, } \\
\text { Jiménez-Sánchez y } \\
\text { Lavín }\end{array}$ & 2018 & $\begin{array}{l}\text { Revista Latina de } \\
\text { Comunicación Social }\end{array}$ \\
\hline 6 & $\begin{array}{l}\text { «Las industrias culturales y creativas en las comunidades autónomas } \\
\text { españolas: el caso Cataluña» }\end{array}$ & $\begin{array}{l}\text { Murciano-Martínez y } \\
\text { González-Saavedra }\end{array}$ & 2018 & $\begin{array}{l}\text { Revista Latina de } \\
\text { Comunicación Social }\end{array}$ \\
\hline 7 & $\begin{array}{l}\text { «Las industrias culturales y creativas en la Comunidad Autónoma del País } \\
\text { Vasco: Características, evolución y distribución espacial» }\end{array}$ & Seco y Gainza & 2018 & $\begin{array}{l}\text { Lurralde: Investigación y } \\
\text { Espacio }\end{array}$ \\
\hline 8 & $\begin{array}{l}\text { «Percepción de valores culturales en trabajadores y su importancia en el } \\
\text { desempeño en la industria maquiladora» }\end{array}$ & $\begin{array}{l}\text { Medrano-López y } \\
\text { Ahumada-Tello }\end{array}$ & 2018 & Contaduría y Administración \\
\hline 9 & $\begin{array}{l}\text { «Ecodiseño en la industria del mueble: oportunidades y desafíos para la } \\
\text { inserción organizativa» }\end{array}$ & $\begin{array}{l}\text { Pereira, D., da Cunha } \\
\text { y Pereira }\end{array}$ & 2018 & Ambiente \& Sociedade \\
\hline 10 & $\begin{array}{l}\text { «Entre neomachismo y retrosexismo: Antifeminismo en industrias } \\
\text { culturales» }\end{array}$ & Menéndez & 2017 & Prisma Social \\
\hline 11 & $\begin{array}{l}\text { «Investigaciones arqueológicas de cazadores-recolectores en el sitio Laguna } \\
\text { Cabeza de Buey } 2 \text { - centro de los pastizales pampeanos, Buenos Aires- } \\
\text { Cincuenta años después de las industrias culturales definidas por Bórmida» }\end{array}$ & Messineo y Scheifler & 2016 & $\begin{array}{l}\text { Intersecciones en } \\
\text { Antropología }\end{array}$ \\
\hline 12 & $\begin{array}{l}\ll \text { Industrias culturales }<\text { AfroPacíficas }>\text { : Encrucijadas del multiculturalismo en } \\
\text { la ciudad de Cali, Colombia» }\end{array}$ & Cárdenas & 2016 & Antípoda \\
\hline 13 & $\begin{array}{l}\text { «Industrias culturales y de la comunicación: mito y lógica de la creatividad y } \\
\text { el empresario innovador» }\end{array}$ & de Mateo-Pérez & 2015 & $\begin{array}{l}\text { Revista Latina de } \\
\text { Comunicación Social }\end{array}$ \\
\hline 14 & $\begin{array}{l}\text { «Por qué el periodismo ciudadano no es una amenaza para la industria de la } \\
\text { prensa: La producción cultural del usuario frente a las industrias culturales» }\end{array}$ & $\begin{array}{l}\text { Pérez-Rufí, Gómez- } \\
\text { Pérez y Navarrete- } \\
\text { Cardero }\end{array}$ & 2015 & $\begin{array}{l}\text { Estudios Sobre el Mensaje } \\
\text { Periodístico }\end{array}$ \\
\hline 15 & «Industrias culturales y estética: un rastreo sobre su posible relación» & Arango-Lopera & 2015 & Palabra Clave \\
\hline 16 & $\begin{array}{l}\text { «Discursos del conocimiento: disyuntivas culturales y sus implicaciones para } \\
\text { las industrias de la lengua» }\end{array}$ & Bennett & 2014 & Ibérica \\
\hline 17 & «Las industrias culturales en español» & Santos y Piedras & 2014 & $\begin{array}{l}\text { Revista Internacional de } \\
\text { Lingüística Iberoamericana }\end{array}$ \\
\hline 18 & $\begin{array}{l}\text { «Industria y agentes culturales: cambios en el paradigma editorial ante la } \\
\text { esfera digital» }\end{array}$ & Montiel & 2014 & Inti \\
\hline 19 & $\begin{array}{l}\text { «Industrias, distritos, instituciones y escenas. Tipología de clústeres } \\
\text { culturales en Barcelona» }\end{array}$ & Ulldemolins y Zarlenga & 2014 & $\begin{array}{l}\text { Revista Española de } \\
\text { Sociología }\end{array}$ \\
\hline 20 & $\ll$ El debate sobre las industrias culturales y creativas » & Echeverría & 2013 & $\begin{array}{l}\text { Cuadernos } \\
\text { Hispanoamericanos }\end{array}$ \\
\hline 21 & $\begin{array}{l}\text { «Una nueva defensa: una biografía cultural de la calidad en la pesca } \\
\text { puertorriqueña» }\end{array}$ & $\begin{array}{l}\text { Griffith, García- } \\
\text { Quijano y Pizzini }\end{array}$ & 2013 & American Anthropologist \\
\hline 22 & «Industrias culturales en ciudades españolas. Un primer acercamiento» & Panal y Yáñez & 2012 & $\begin{array}{l}\text { Revista de Estudios } \\
\text { Regionales }\end{array}$ \\
\hline 23 & $\begin{array}{l}\text { «De las industrias culturales a las industrias del ocio y las creativas: los límites } \\
\text { del campo cultural» }\end{array}$ & Ferrándiz & 2011 & Comunicar \\
\hline 24 & «Las industrias culturales y de información: un enfoque socioeconómico» & Miège & 2008 & $\begin{array}{l}\text { Revista Electrónica de } \\
\text { Investigación Educativa }\end{array}$ \\
\hline 25 & «Las industrias culturales y los contenidos de internet en español» & Lafuente & 2000 & Arbor \\
\hline
\end{tabular}

Fuente. Elaboración propia con base en Scopus. 
A partir de lo anterior se logró identificar que España es el país con una mayor cantidad de revistas que han publicado investigaciones sobre el tema de estudio y bajo el descriptor utilizado, con una participación de aproximadamente el $34 \%$ sobre el total de estas, lo cual demuestra mayor avance y mayor cobertura del tema cultural.

Tabla 2. Índice de impacto y país de origen

\begin{tabular}{|l|l|l|}
\hline \multicolumn{1}{|c|}{ Revista } & \multicolumn{1}{c|}{ País de origen } & SJR (2018) \\
\hline American Anthropologist & Estados Unidos & 1,026 \\
\hline Antípoda & Colombia & 0,131 \\
\hline Arbor & España & 0,153 \\
\hline Comunicar & España & 0,851 \\
\hline Contaduría y Administración & México & 0,238 \\
\hline Cuadernos Hispanoamericanos & España & 0,110 \\
\hline Estudios Sobre el Mensaje Periodístico & España & 0,232 \\
\hline Ibérica & Portugal & 0,259 \\
\hline Información Tecnológica & Chile & 0,210 \\
\hline Intersecciones en Antropología & Argentina & 0,420 \\
\hline Inti & España & 0,101 \\
\hline Kepes & Colombia & 0,208 \\
\hline Lurralde: Investigación y Espacio & España & 0,106 \\
\hline Palabra Clave & Colombia & 0,260 \\
\hline Prisma Social & España & 0,157 \\
\hline Canadian Journal of Latin American and Caribbean Studies & Canadá & 0,148 \\
\hline Revista de Estudios Regionales & España & 0,122 \\
\hline Revista Electrónica de Investigación Educativa & México & 0,431 \\
\hline Revista Española de Sociología & España & 0,255 \\
\hline Revista Internacional de Lingüística Iberoamericana & España & 0,342 \\
\hline International Journal of Cultural Policy & Estados Unidos & 0,475 \\
\hline Revista Latina de Comunicación Social & España & 0,509 \\
\hline & & \\
\hline
\end{tabular}

Fuente. Elaboración propia con base en Scopus.

En cuanto a países latinoamericanos, Brasil se encuentra en el top, pues abarca el $21 \%$ de revistas, en la medida en que posee mayor cantidad de artículos relacionados. Adicionalmente, es válido resaltar que Colombia posee, en este estudio, el $15 \%$ de las revistas, un porcentaje positivo para la balanza realizada por número de revistas, debido a que se trata de una serie de temáticas culturales que dentro del país tienen aún un tinte novedoso (véase la Tabla 2). 
Ahora bien, en su mayoría, se trató de journals que fueron indexadas por Scopus a partir del año 2008, periodo en el que se empieza a reconstruir un mayor número de artículos vinculados a la temática de las industrias culturales, siendo en su mayoría estudios de caso y temas de comunidades específicas, que de alguna forma se empiezan a relacionar con la cultura y con el ecosistema creativo global, demostrando que la aparición del descriptor utilizado se empieza a relacionar desde particularidades socio-culturales propias.

Ahora bien, en cuanto a las áreas temáticas, se logró hallar que la mayor cantidad de revistas analizadas, dentro de sus áreas temáticas o categorías afines a las ICC, contenían el área denominada «artes y humanidades: historia», ya que varios de los artículos analizan los efectos del cine en los años setenta del siglo XX y la música tradicional en pueblos indígenas, entre otros. Otra área a resaltar, la cual tuvo gran participación, es «artes y humanidades: artes visuales y artes escénicas», que, como su nombre lo indica, está presente en artículos cuyos enfoques son el teatro, la danza, el grafiti o los jeroglíficos.

Respecto al índice de impacto, aún se trata de cifras bajas (véase la Tabla 2), si se tiene en cuenta el periodo de tiempo de medición, dado que se trata de una selección de journals con temática particular, de modo que se demuestra la necesidad de mayores investigaciones que referencien este tópico en términos generales.

\section{Discusión}

\begin{abstract}
A partir dela revisión realizada y bajo el descriptor 1 seleccionado, los resultados se configuran a partir de las temáticas identificadas y el impacto que generó cada uno de los artículos. Esto demuestra que, si bien los resultados en cantidad son bajos, el impacto que han generado estas especificidades, las cuales hacen parte del sector cultural, se tornan importantes y se establecen como punto de partida para futuras investigaciones.
\end{abstract}

De esta manera, se logró identificar que las temáticas, aunque se relacionan con el tópico, no responden, en su totalidad, a estudios representativos del desarrollo de este tipo de industrias, sino que se han enfocado en lineamientos específicos y a grupos humanos particulares, lo cual atribuye la temática a especificidades que no se relacionan con el total de la comunidad. Es decir, que los artículos seleccionados presentan temáticas relacionadas con las industrias culturales a partir de su vinculación con un grupo de personas particulares, de modo que es este elemento vinculador y determinante para la presentación del tema. Lo anterior deja sobre la mesa $-y$ con miras a futuras reflexiones - la necesidad de integrar al total de la sociedad civil en investigaciones que se lideren desde la academia.

A continuación, se especifica la problematización de los artículos encontrados, teniendo en cuenta que más allá de la descripción de cada uno se trata de la agrupación de temáticas y el alcance de cada una con respecto a la concepción y al reconocimiento del concepto de industrias culturales que, al final, se convierte en una guía de investigación. 
Tabla 3. Problematización del concepto industrias culturales

\begin{tabular}{|c|c|}
\hline Artículos IC-revistas & Problematización de las IC \\
\hline $\begin{array}{l}\text { Arbor } \\
\text { Revista Latina de Comunicación Social } \\
\text { Estudios Sobre el Mensaje Periodístico } \\
\text { Palabra Clave } \\
\text { Kepes } \\
\text { Palabra Clave }\end{array}$ & $\begin{array}{l}\text { IC: como fuentes de información, representadas desde categorías } \\
\text { de intermediación. } \\
\text { Como parte de un proceso de comunicación entre diversos } \\
\text { actores. }\end{array}$ \\
\hline $\begin{array}{l}\text { Antípoda } \\
\text { Intersecciones en Antropología } \\
\text { Revista de Estudios Regionales }\end{array}$ & $\begin{array}{l}\text { IC: como representantes de grupos sociales específicos. Le atri- } \\
\text { buyen una misión al concepto para difundir una idea o un valor } \\
\text { específico asociado a una comunidad }\end{array}$ \\
\hline Revista Electrónica de Investigación Educativa & $\begin{array}{l}\text { IC: como concepto que naturalmente mezcla el aspecto econó- } \\
\text { mico a su desarrollo y que vincula un elemento comercial. }\end{array}$ \\
\hline $\begin{array}{l}\text { Prisma Social } \\
\text { American Anthropologist } \\
\text { Cuadernos Hispanoamericanos } \\
\text { Revista Española de Sociología } \\
\text { Inti } \\
\text { Revista Internacional de Lingüística Iberoamericana } \\
\text { Ibérica } \\
\text { Canadian Journal of Latin American and Caribbean Studies } \\
\text { Revista Latina de Comunicación Social } \\
\text { Lurralde: Investigación y Espacio }\end{array}$ & $\begin{array}{l}\text { IC: concepto utilizado para referirse a la identidad, como repre- } \\
\text { sentante de elementos diferenciadores de grupo y como meca- } \\
\text { nismo de desarrollo de identificación. }\end{array}$ \\
\hline $\begin{array}{l}\text { International Journal of Cultural Policy } \\
\text { Revista Latina de Comunicación Social } \\
\text { Información Tecnológica } \\
\text { Ambiente \& Sociedade } \\
\text { Comunicar }\end{array}$ & $\begin{array}{l}\text { IC: como generadoras de procesos innovadores. El concepto } \\
\text { se utilizó para agrupar sectores o casos específicos en los que la } \\
\text { creatividad intervino y generó oportunidades económicas. }\end{array}$ \\
\hline
\end{tabular}

Fuente. Elaboración propia con base en Scopus.

Se debe decir que la aparición del concepto en la base de datos Scopus se da en el 2008 —en conformidad con la búsqueda planteada - a excepción de un único paper que aparece en el 2000, «Las industrias culturales y los contenidos de internet en español» (Lafuente, 2000), el cual desarrolla una temática de uno de los subsectores que hace parte del sector cultural. Así, se presenta en esta primera aparición el concepto en cuanto macro sector que vincula la difusión necesaria de contenidos y se establece de esta forma a las industrias culturales como intermediarias de información, sin reconocer, aún, la diversidad interna que posee. Sin embargo, sí como un punto de referencia y una primera mención que logró verse de manera general con posibilidad de explorarse y diversificarse.
En los años siguientes, y hasta el 2007, no se encontraron resultados para la búsqueda planteada, mas es en el 2008 de nuevo aparece el descriptor, bajo el paper «Las industrias culturales y de información: un enfoque socioeconómico» (Miège, 2008) en el que el concepto adquiere una característica de comercialización, es decir, no solo se plantea como dinamizador de cultura, sino que se mezcla con la disciplina económica al plantear la necesidad de tratar a los productos culturales con valor comercial capaces de entrar en un mercado y establecer alianzas con grupos financieros; con esto se le otorga un carácter consumista. Según Adorno (1987), los productos resultantes de la industria cultural se consideran parte de una sociedad de consumo y poseen valor desde su utilización y homogeneización 
de la sociedad, de manera que es la primera teoría reconocida del concepto de industria cultural, desde la visión consumista.

Para el 2011, el paper «De las industrias culturales a las industrias del ocio y las creativas: los límites del campo cultural» (Ferrándiz, 2011) presenta a las industrias culturales como parte del ocio, de la recreación y hasta de la creatividad. Esto implica que se desdibujan los límites entre cultura y entretenimiento, de modo que se dejan de lado las características de trabajo del campo cultural y se sitúan como parte de una evolución tecnológica que facilita su uso con fines recreativos.

En el 2012 se plantea una identificación del sector a través del paper titulado «Industrias culturales en ciudades españolas. Un primer acercamiento» (Panal y Yáñez, 2012). En este trabajo el concepto se torna relevante para el desarrollo de poblaciones o territorios posindustriales, de manera que se convierten en ciudades creativas. Esto lleva a reconocer a las industrias culturales como catalizadoras de desarrollo urbano, en cuanto intervienen en la estructuración de nuevos modelos de desarrollo.

Para el 2013 se encuentran dos investigaciones: «El debate sobre las industrias culturales y creativas» (Echeverría, 2013) y «Una nueva defensa: una biografía cultural de la calidad en la pesca puertorriqueña» (Griffith et al., 2013), en las cuales el concepto empieza a desarrollarse como parte de estudios de caso y se plantea como facilitador de identidad, en la medida en que son responsables de la identificación de características particulares de grupos específicos de personas. Es en este punto que en las investigaciones encontradas empieza a reconocerse su papel como garantes de identidad.

Cuatro investigaciones se encuentran durante el 2014, con lo que se manifiesta el incremento de investigaciones sobre cultura. Estas fueron: «Discursos del conocimiento: disyuntivas culturales y sus implicaciones para las industrias de la lengua» (Bennett, 2014), «Las industrias culturales en español» (Santos y Piedras, 2014), «Industria y agentes culturales: cambios en el paradigma editorial ante la esfera digital» (Montiel, 2014) e «Industrias, distritos, instituciones y escenas. Tipología de clústeres culturales en Barcelona» (Ulldemolins y Zarlenga, 2014). Cada una manifiesta la importancia de las industrias culturales como parte de vinculante de poblaciones específicas, con características propias y elementos identitarios propios; igualmente, se reconoce la transformación del concepto, al presentarlo en una relación con la economía y la necesidad de medir su contribución a la sociedad, punto que ha sido necesario pero escaso hasta el día de hoy en Latinoamérica.

En el 2015, los papers encontrados, «Industrias culturales y de la comunicación: mito y lógica de la creatividad y el empresario innovador» (de MateoPérez, 2015) y «Por qué el periodismo ciudadano no es una amenaza para la industria de la prensa: la producción cultural del usuario frente a las industrias culturales» (Pérez-Rufí et al., 2015), así como «Industrias culturales y estética: un rastreo sobre su posible relación» (Arango-Lopera, 2015), atribuyen a las industrias culturales un carácter innovador, una característica propia de quienes son protagonistas del sector cultural con la que vinculan una perspectiva estética de los que realizan contenidos culturales. Así, con la innovación, las industrias culturales permiten que sus protagonistas o integrantes planteen soluciones con estilos diversos y se sitúen como proveedores de contenido cultural.

En el 2016, dos investigaciones, «Investigaciones arqueológicas de cazadores-recolectores en el sitio Laguna Cabeza de Buey 2-centro de los pastizales pampeanos, Buenos Aires-. Cincuenta años después de las industrias culturales definidas por Bórmida» (Messineo y Scheifler, 2016) e «Industrias $<$ AfroPacíficas >: encrucijadas del multiculturalismo en la ciudad de Cali, Colombia» (Cárdenas, 2016), ejemplifican a las industrias culturales como sectorizadas, como el conjunto de actividades que están diseñadas por y para grupos específicos, y que los beneficios, por ende, van dirigidos a ciertos sectores de la sociedad. En este punto resulta interesante descubrir que, si bien son generadoras de desarrollo económico o de reconocimiento social, están dadas a 
partir de prácticas socialmente excluyentes, según los estudios presentados.

Durante el 2017, el paper «Entre neomachismo y retrosexismo: antifeminismo en industrias culturales» (Menéndez, 2017) presenta una transformación más dentro del concepto trabajado, de tal manera que le brinda un papel representativo para la reivindicación de los derechos de la mujer, y expone así la necesaria configuración de espacios culturales como garantes de equidad y catalizadores de oportunidades para la libertad de las mujeres.

Finalmente, durante el 2018 se encontró la mayoría de artículos representativos del descriptor utilizado, de modo que se generaron varias transformaciones al concepto y se delimitaron las investigaciones más recientes. Esto demuestra el incremento en el interés investigativo frente a un sector que actualmente aún se desarrolla de manera diversa pero significativa con miras a la estructuración y el pleno conocimiento del accionar de sus protagonistas.

En primer lugar, se presenta a las industrias culturales como medios que generan oportunidades en comunidades específicas, así como agrupaciones que presentan características particulares de grupos de personas; de igual modo, como exclusivas, en términos de peculiaridades y beneficios.

Esto mediante los siguientes artículos: «La invención del cocacolo: <americanización> y diferenciación social en Bogotá en la década de 1950» (Flórez-
Malagón, 2018), «Industrias culturales y composición de los personajes en las series de animación infantil emitidas en España» (OrtegaMohedano et al., 2018), «Percepción de valores culturales en trabajadores y su importancia en el desempeño en la industria maquiladora» (MedranoLópez y Ahumada-Tello, 2018) y «Ecodiseño en la industria del mueble: oportunidades y desafíos para la inserción organizativa» (Pereira et al., 2018).

En segundo lugar, durante el 2018, el concepto de industrias culturales fue exteriorizado a través de su dinamismo dentro de la economía de un Estado, es decir, se trata de un ecosistema que se nutre de ventajas tecnológicas y que se ha convertido en líder de desarrollo social y económico; que además, necesita regulación con el fin de obtener derechos culturales. Los artículos que presentan esta configuración fueron «Innovaciones en política cultural y desarrollo en América Latina» (Yúdice, 2018), «Industrias creativas y culturales: estudio desde el enfoque de la gestión del conocimiento» (Blanco-Valbuena et al., 2018), «Perspectivas investigativas en el estudio de las industrias culturales y creativas» (Avendaño et al., 2018), «Las industrias culturales y creativas en las comunidades autónomas españolas: el caso Cataluña» (Murciano-Martínez y González-Saavedra, 2018) y «Las industrias culturales y creativas en la Comunidad Autónoma del País Vasco: características, evolución y distribución espacial» (Seco y Gainza, 2018).

Tabla 4. Transformación y uso del concepto industrias culturales desde la base Scopus —2000-2018_

\begin{tabular}{|c|c|}
\hline Año & Transformación \\
\hline 2000 & IC como intermediarias de información \\
\hline 2008 & IC como producto comercializable \\
\hline 2011 & IC como entretenimiento \\
\hline 2012 & IC como catalizadoras de desarrollo urbano \\
\hline 2013 & IC como facilitadoras de identidad \\
\hline 2014 & IC como representantes de poblaciones específicas \\
\hline 2015 & IC como innovación \\
\hline 2016 & IC socialmente excluyentes \\
\hline 2017 & IC como medios de defensa de derechos \\
\hline 2018 & IC como garantes de beneficios sectorizados y como parte de un ecosistema creativo que se debe regular \\
\hline
\end{tabular}

Fuente. Elaboración propia a partir de Scopus. 


\section{Conclusiones}

as industrias culturales, toman de manera
progresiva cada vez más relevancia como objeto de estudio e investigación dentro de la academia debido a su incursión en varios sectores de la vida social y cultural de las personas de un grueso social. Esto en cuanto a que, por un lado, «permiten la apropiación de elementos identitarios con el fin de ser reproducidos y difundidos como garantes de representación y de identificación, en un contexto globalizado desprovisto de prioridades sociales y culturales $\gg$ (Redondo-Méndez,2019,p.42), de modo que son representantes de identidades y tradiciones; y por otro, como espacios de investigación que permiten la inclusión de novedosas temáticas tratadas a partir del reconocimiento del sector cultural.

Sin embargo, es necesario adelantar aún más la investigación para encontrar datos como, por ejemplo, la necesidad de información que tiene Colombia en este tema, a fin de lograr aportar de forma significativa en las mejoras que requieren estas industrias $y$, sobre todo, para que grupos de investigación, entidades y particulares interesados en conocer o estudiar aún más la economía creativa logren tener con mayor facilidad un insumo como el estado de arte que aquí se realiza; para que este sea la base del estudio teórico que requieren para adelantar su distintas investigaciones.

Por lo anterior, esta fase investigativa permitió generar un aporte significativo al conocimiento en torno a las industrias culturales, el cual aprovechará a futuro no solo el actual grupo investigador, sino los futuros investigadores que se interesen también por analizar el sector cultural, dado que mostrará, en una primera fase, los temas más favorecidos para estas publicaciones y los menos estudiados. Se trató de una aproximación al uso del concepto de industrias culturales en el marco de investigaciones de impacto y en un contexto espacio-temporal seleccionado.

De la misma manera, el uso conceptual evidencia el avance de la percepción que desde el 2000 se ha generado en la academia a nivel global, si se tiene en cuenta que el concepto aparece, por primera vez, dentro de investigaciones de tipo académico y en la base de datos seleccionada en este año.

Lo anterior implica que hablar de industrias culturales supone el entendimiento de un sector que vincula a determinados grupos poblacionales y genera ventajas para estos, pero que no ha logrado vincular al total de la población, en términos investigativos, debido a que resaltan casos específicos de estudio y su éxito en el desarrollo, ya sea de nuevas tecnologías o en la vinculación e inclusión social. Esto demuestra que, aun cuando se reconoce plenamente, dentro del ecosistema creativo global actual, que para que funcione de manera efectiva la labor cultural debe intervenir la totalidad de los actores de un territorio, directos o indirectos, hace falta emprender acciones que realmente garanticen dicha participación.

La aproximación conceptual a la que se hace referencia con esta primera fase investigativa se entiende en términos de vinculación de nuevas temáticas en las que las industrias culturales pueden ingresar $y$ en las que pueden generar mayores espacios de accionar en sociedades que adolecen de la capacidad de garantizar y proveer un bienestar por parte del Estado. La transformación del concepto, en términos investigativos -y en los límites planteados- se ha desarrollado a partir de experiencias específicas que abren espacios novedosos de intervención y procesos investigativos que aporten no solo con casos de estudio, sino también con la construcción de estructuras y evaluación de datos.

Así, se presenta un recorrido de 18 años que demuestra su aparición como intermediarias de información, hasta un sector que se que presenta a las industrias culturales como intermediarias de información, o incluso como un sector que se maneja bajo sus propias reglas y que requiere una regulación por parte del Estado a fin de lograr crear políticas culturales que reconozcan sus fortalezas y que abran espacios de difusión. Por lo tanto, con la 
búsqueda realizada se puede establecer que quedan aún vacíos frente a los apoyos que desde el Estado se necesitan, con énfasis en el caso latinoamericano, pues se vieron, desde el año 2008, casos particulares de estudio en los que se desarrollaron subsectores culturales bajo modalidades propias, de trabajo y éxito sustentado en la innovación.

La integración de diversas y variadas disciplinas dentro del campo de estudio de las industrias culturales es uno de los resultados más representativos, en la medida en que amplía el reconocimiento del concepto y no lo limita a un sector en particular; por el contrario, abre su dimensión investigativa y la percepción social que de él se tiene, con el fin de lograr estudios representativos desde otras disciplinas. Esto significa, entonces, que se convierte en un derrotero interdisciplinar y multidisciplinar para nuevos temas de investigación en Colombia que vinculen a las industrias culturales.
Lo anterior lleva al reconocimiento de las industrias culturales como un espacio investigativo capaz de integrar metodologías interdisciplinarias variadas, con un alto impacto en el campo investigativo, dada su capacidad de relacionar el sector cultural con ámbitos y esferas sociales que impactan en el desarrollo de un Estado. Así, no solo se reconoce la categoría de artes y humanidades, desde aspectos tales como la lingüística o las artes, sino que se vincula con las ciencias sociales desde la cultura, la educación, la comunicación, la sociología y las ciencias políticas, hasta llegar a los negocios internacionales y la administración.

Esto se debe convertir en un primer paso para el acercamiento a un espacio bastante amplio de investigación que, como fue posible evidenciar, presenta escenarios diversos de transformación social y económica, y brinda, además, oportunidades de generación de conocimiento basadas tanto en experiencias de grupos poblacionales específicos como en los aportes académicos de diferentes disciplinas. 


\section{Referencias}

Adorno, T. (1987). La industria cultural. Buenos Aires: Galerna.

Arango-Lopera, C. A. (2015). Industrias culturales y estética: un rastreo sobre su posible relación. Palabra Clave, 18(2), 499536. DOI: https://doi.org/10.5294/pacla.2015.18.2.8

Avendaño, C. H.; Arias, A. V.; Arias, M. B. (2018). Perspectivas investigativas en el estudio de las industrias culturales y creativas. Kepes, 15(17), 27-67.

Bennett, K. (2014). Discursos del conocimiento: disyuntivas culturales y sus implicaciones para las industrias de la lengua. Ibérica, 27, 35-49.

Blanco-Valbuena, C. E.; Bernal-Torres, C. A.; Camacho, F.; DíazOlaya, M. (2018). Industrias creativas y culturales: estudio desde el enfoque de la gestión del conocimiento. Información Tecnológica, 29(3), 15-28. DOI: https://doi.org/10.4067/ S0718-07642018000300015

Cárdenas, M. P. (2016). Industrias culturales «AfroPacíficas»: encrucijadas del multiculturalismo en la ciudad de Cali, Colombia. Antípoda, (24), 75-90. DOI: https://doi. org/10.7440/antipoda24.2016.05

de Mateo-Pérez, R. de. (2015). Industrias culturales y de la comunicación: mito y lógica de la creatividad y el empresario innovador. Revista Latina de Comunicación Social, (70), 813832. DOI: https://doi.org/10.4185/RLCS-2015-1073

Echeverría, J. (2013). El debate sobre las industrias culturales y creativas. Cuadernos Hispanoamericanos, (761), 21-33.

EY. (2015). Tiempos de cultura. El primer mapa mundial de las industrias culturales y creativas. Cisac. Recuperado de https:// bit.ly/3wy0MbY

Ferrándiz, R. R. (2011). De las industrias culturales a las industrias del ocio y las creativas: los límites del campo cultural. Comunicar, 18(36), 149-156. DOI: https://doi. org/10.3916/C36-2011-03-06

Flórez-Malagón, A. G. (2018). La invención del cocacolo: «americanización»y diferenciación social en Bogotá en la década de 1950. Canadian Journal of Latin American and Caribbean Studies, 43(3), 315-336. DOI: https://doi.org/10 $.1080 / 08263663.2018 .1486951$

Follari, R. (2003). Adorno y Benjamin sobre la cultura: acerca de un equívoco persistente. Revista Confluencia, 1(3), 83-108. Recuperado de https://bit.ly/3cYoqqr

Griffith, D.; García-Quijano, C.; Pizzini, M. V. (2013). Una nueva defensa: una biografía cultural de la calidad en la pesca puertorriqueña. American Anthropologist, 115(1), 17-28. DOI: https://doi.org/10.1111/j.1548-1433.2012.01532.x

Horkheimer, M.; Adorno, T. W. (1988). Dialéctica del iluminismo. Buenos Aires: Sudamericana.
Howkins, J. (2001). The creative economy. Londres: Penguin Books.

Lafuente, F. R. (2000). Las industrias culturales y los contenidos de internet en español. Arbor, 167(658), 325-335. DOI: https://doi.org/10.3989/arbor.2000.1658.1165

Medrano-López, D.; Ahumada-Tello, E. (2018). Percepción de valores culturales en trabajadores y su importancia en el desempeño en la industria maquiladora. Contaduría $y$ Administración, 63(3), 1-30. DOI: https://doi.org/10.22201/ fca.24488410e.2018.1264

Menéndez, M. I. (2017). Entre neomachismo y retrosexismo: antifeminismo en industrias culturales. Prisma Social, (NE 2), 1-30. Recuperado de https://bit.ly/3wy11DU

Messineo, P. G.; Scheifler, N. A. (2016). Investigaciones arqueológicas de cazadores-recolectores en el sitio Laguna Cabeza de Buey 2 - centro de los pastizales pampeanos, Buenos Aires-. Cincuenta años después de las industrias culturales definidas por Bórmida. Intersecciones en Antropología, 17(2), 213-226. Recuperado de https://bit.ly/31Vkpgb

Miége, B. (2008). Las industrias culturales y de información: un enfoque socioeconómico. Revista Electrónica de Investigación Educativa, 10(1), 1-15. Recuperado de https://bit. ly/3sX2PnP

Montiel, D. E. (2014). Industria y agentes culturales: cambios en el paradigma editorial ante la esfera digital. Inti, 79(80), 215234.

Murciano-Martínez, M.; González-Saavedra, C. (2018). Las industrias culturales y creativas en las comunidades autónomas españolas: el caso Cataluña. Revista Latina de Comunicación Social, (73), 146-167. DOI: https://doi.org/10.4185/RLCS2018-1250

Ortega-Mohedano, F.; Jiménez-Sánchez, A.; Lavín, J. M. (2018). Industrias culturales y composición de los personajes en las series de animación infantil emitidas en España. Revista Latina de Comunicación Social, (73), 74-85. DOI: https://doi. org/10.4185/RLCS-2018-1246

Panal, G. G.; Yáñez, C. N. (2012). Industrias culturales en ciudades españolas. Un primer acercamiento. Revista de Estudios Regionales, (94), 71-103.

Pereira, D.; da Cunha, S. K.; Pereira, L. (2018). Ecodiseño en la industria del mueble: oportunidades y desafíos para la inserción organizativa. Ambiente \& Sociedade, (21), E00791.

Pérez-Rufí, J. P.; Gómez-Pérez, F. J.; Navarrete-Cardero, J. L. (2015). Por qué el periodismo ciudadano no es una amenaza para la industria de la prensa: la producción cultural del usuario frente a las industrias culturales. Estudios Sobre el Mensaje Periodístico, 21(2), 899-912. DOI: https://doi.org/10.5209/ rev_ESMP.2015.v21.n2.50891 
Redondo-Méndez, A. C. (2019). Industrias culturales latinoamericanas y su aporte al desarrollo social. En. J. Hernández-Acosta; A. C. Redondo-Méndez; O. Ospina Martínez (Eds.) Industrias culturales y economía creativa en Latinoamérica. Desarrollo económico y social en la región. (2370). Bogotá: Editorial Uniagustiniana. DOI: https://doi. org/10.28970/9789585498143.1

Santos, M; Piedras, E. (2014). Las industrias culturales en español. Revista Internacional de Lingüística Iberoamericana, 12(24), 87-103.

Seco, E.; Gainza, X. (2018). Las industrias culturales y creativas en la Comunidad Autónoma del País Vasco: características, evolución y distribución espacial. Lurralde: Investigación y Espacio, (41), 33-53.

SJR (Scimagojr). (2018). Scimago Journal \& Country Rank. Recuperado de http://www.scimagojr.com/journalrank.php

Torraco, R. J. (2005). Writing Integrative Literature Reviews: Guidelines and Examples. Human Resource Development Review, 4(3), 356-367. DOI: https://doi. org/10.1177/1534484305278283

Ulldemolins, J. R.; Zarlenga, M. I. (2014). Industrias, distritos, instituciones y escenas. Tipología de clústeres culturales en Barcelona. Revista Española de Sociología, (21), 47-68. Recuperado de https://bit.ly/2Rnyq4l

Yúdice, G. (2018). Innovaciones en política cultural y desarrollo en América Latina. International Journal of Cultural Policy, 24(5), 647-663. DOI: https://doi.org/10.1080/10286632.2 018.1514034 